\title{
Optical Coherence Tomography Angiography Features of Iris Racemose Hemangioma in 4 Cases.
}

\author{
Jason L. Chien \\ Thomas Jefferson University \\ Kareem Sioufi \\ Thomas Jefferson University \\ Sandor Ferenczy \\ Thomas Jefferson University \\ Emil Anthony T. Say \\ Thomas Jefferson University \\ Carol L. Shields \\ Thomas Jefferson University \\ Follow this and additional works at: https://jdc.jefferson.edu/willsfp \\ Part of the Ophthalmology Commons \\ Let us know how access to this document benefits you
}

\section{Recommended Citation}

Chien, Jason L.; Sioufi, Kareem; Ferenczy, Sandor; Say, Emil Anthony T.; and Shields, Carol L., "Optical Coherence Tomography Angiography Features of Iris Racemose Hemangioma in 4 Cases." (2017). Wills Eye Hospital Papers. Paper 76.

https://jdc.jefferson.edu/willsfp/76

This Article is brought to you for free and open access by the Jefferson Digital Commons. The Jefferson Digital Commons is a service of Thomas Jefferson University's Center for Teaching and Learning (CTL). The Commons is a showcase for Jefferson books and journals, peer-reviewed scholarly publications, unique historical collections from the University archives, and teaching tools. The Jefferson Digital Commons allows researchers and interested readers anywhere in the world to learn about and keep up to date with Jefferson scholarship. This article has been accepted for inclusion in Wills Eye Hospital Papers by an authorized administrator of the Jefferson Digital Commons. For more information, please contact: JeffersonDigitalCommons@jefferson.edu. 


\section{Optical Coherence Tomography Angiography Features of Iris Racemose Hemangioma in 4 Cases}

Jason L. Chien, BS; Kareem Sioufi, MD; Sandor Ferenczy, CRA, OCT-C; Emil Anthony T. Say, MD;

Carol L. Shields, MD

IMPORTANCE Optical coherence tomography angiography (OCTA) allows visualization of iris racemose hemangioma course and its relation to the normal iris microvasculature.

OBJECTIVE To describe OCTA features of iris racemose hemangioma.

DESIGN, SETTING, AND PARTICIPANTS Descriptive, noncomparative case series at a tertiary referral center (Ocular Oncology Service of Wills Eye Hospital). Patients diagnosed with unilateral iris racemose hemangioma were included in the study.

MAIN OUTCOMES AND MEASURES Features of iris racemose hemangioma on OCTA.

RESULTS Four eyes of 4 patients with unilateral iris racemose hemangioma were included in the study. Mean patient age was 50 years, all patients were white, and Snellen visual acuity was 20/20 in each case. All eyes had sectoral iris racemose hemangioma without associated iris or ciliary body solid tumor on clinical examination and ultrasound biomicroscopy. By anterior segment OCT, the racemose hemangioma was partially visualized in all cases. By OCTA, the hemangioma was clearly visualized as a uniform large-caliber vascular tortuous loop with intense flow characteristics superimposed over small-caliber radial iris vessels against a background of low-signal iris stroma. The vascular course on OCTA resembled a light bulb filament (filament sign), arising from the peripheral iris (base of light bulb) and forming a tortuous loop on reaching its peak (midfilament) near the pupil ( $n=3$ ) or midzonal iris $(n=1)$, before returning to the peripheral iris (base of light bulb). Intravenous fluorescein angiography performed in 1 eye depicted the iris hemangioma; however, small-caliber radial iris vessels were more distinct on OCTA than intravenous fluorescein angiography.

CONCLUSIONS AND RELEVANCE Optical coherence tomography angiography is a noninvasive vascular imaging modality that clearly depicts the looping course of iris racemose hemangioma. Optical coherence tomography angiography depicted fine details of radial iris vessels, not distinct on intravenous fluorescein angiography.

JAMA Ophthalmol. 2017;135(10):1106-1110. doi:10.1001/jamaophthalmol.2017.3390 Published online September 14, 2017.

\author{
Author Affiliations: Ocular Oncology \\ Service, Wills Eye Hospital, Thomas \\ Jefferson University, Philadelphia, \\ Pennsylvania. \\ Corresponding Author: Carol L. \\ Shields, MD, Ocular Oncology \\ Service, Wills Eye Hospital, Thomas \\ Jefferson University, 840 Walnut St, \\ 14th Floor, Philadelphia, PA 19107 \\ (carolshields@gmail.com).
}

ris racemose hemangioma is a benign arteriovenous malformation with direct artery to vein communication but without intervening capillary bed. ${ }^{1,2}$ Reports have used intravenous fluorescein angiography (IVFA), anterior segment (AS) optical coherence tomography (OCT), and ultrasound biomicroscopy (UBM) to describe features of this iris abnormality. ${ }^{3}$ Optical coherence tomography angiography (OCTA) was introduced in the clinical setting in 2012 and is best known for detecting retinal neovascularization, with only limited reports of AS vasculature. ${ }^{4-8}$ We report herein AS imaging of iris racemose hemangioma using OCTA.

\section{Methods}

This is a retrospective observational case series of 4 consecutive unilateral iris racemose hemangiomas seen on the Ocular Oncology Service of Wills Eye Hospital, Philadelphia, Pennsylvania, from August 2016 to November 2016. Institutional review board approval of Wills Eye Hospital was obtained, and Health Insurance Portability and Accountability Act guidelines were followed. Written consent was obtained from all patients. 
All patients underwent comprehensive ophthalmic examination including slitlamp evaluation and photography, dilated funduscopic examination, UBM, AS-OCT, IVFA (when possible), and OCTA. Optical coherence tomography angiography was performed with Optovue RTVue XR Avanti, version 2016.1.0.26 (Optovue Inc). ${ }^{9}$ For AS OCTA, a volume cube scan protocol was used to scan the lesion $(3.00 \times 3.00 \mathrm{~mm}$; AngioRetina); however, the Z-motor positioning and focus settings were adjusted manually to allow visualization of the AS and precise focus of the OCT B-scan, and the P-motor was automatically adjusted.

Patient demographics (including age, race/ethnicity, and sex) and referral diagnoses were recorded. Data regarding clinical features included affected eye, visual acuity, iris color, tumor clock-hour location, tumor size (number of clock hours), and presence of iris or ciliary body mass. Imaging features were recorded from UBM, AS-OCT, IVFA, and OCTA, including visualization of hemangioma, vascular course, and hemangioma type (simple vs complex). ${ }^{10}$

\section{Results}

Mean age was 50 years (range, 43-67 years), and all patients were white with bilateral 20/20 visual acuity. By slitlamp biomicroscopy, the hemangioma was minimally visible within the iris stroma as a large, dark-red vessel with a tortuous course buried within normal stromal tissue without solid tumor in the iris or ciliary body. The hemangioma was located temporally or inferotemporally in the right $(n=3)$ or left $(n=1)$ eye and demonstrated 1.0-1.5 clock hours of involvement (Table).

\section{Key Points}

Question How does iris racemose hemangioma appear by optical coherence tomography angiography?

Finding In this case series using optical coherence tomography angiography, iris racemose hemangioma appears as a large-caliber vascular tortuous loop with intense flow characteristics superimposed over small-caliber radial iris vessels against a background of low-signal iris stroma. It resembles a light bulb filament (filament sign), arising from the peripheral iris (base of light bulb), forming a tortuous loop on reaching its peak (midfilament) near the pupil or midzonal iris, before returning to the peripheral iris (base of light bulb)

Meaning These findings suggest iris racemose hemangioma has distinct features on optical coherence tomography angiography.

On UBM, the hemangioma could not be identified. Anterior segment OCT over the hemangioma showed its lumen as a hyporeflective spherical core with poorly delineated margins against normal iris stroma and vasculature. By OCTA, the hemangioma appeared as a uniform, large-caliber vascular tortuous loop without aneurysmal changes, and with intense flow characteristics superimposed over smallcaliber radial iris vessels against a background of low-signal iris stroma. The vascular course on OCTA resembled a light bulb filament (filament sign), arising from the peripheral iris (light bulb base) and forming a tortuous loop on reaching its peak (midfilament) near the pupil ( $n=3$, Figure $1 B$ and $D$ and Figure $2 \mathrm{D}$ ) or midzonal iris $(\mathrm{n}=1$, Figure $1 \mathrm{~F})$, before returning to the peripheral iris (light bulb base). Optical coherence tomography angiography was not able to visual-

\begin{tabular}{|c|c|c|c|c|}
\hline Demographics & Patient 1 & Patient 2 & Patient 3 & Patient 4 \\
\hline Age, y & 45 & 43 & 43 & 67 \\
\hline Race/ethnicity & White & White & White & White \\
\hline Sex & Female & Male & Male & Female \\
\hline Referral diagnosis & Iris lesion & Iris lesion & Iris lesion & Iris lesion \\
\hline \multicolumn{5}{|l|}{ Clinical features } \\
\hline Involved eye & Right & Right & Right & Left \\
\hline Visual acuity & $20 / 20$ & $20 / 20$ & $20 / 20$ & $20 / 20$ \\
\hline Iris color & Blue & Blue & Green & Green \\
\hline Clock hours, h:min & $7: 00-8: 30$ & $7: 00-8: 30$ & 9:00-10:00 & $4: 00-5: 30$ \\
\hline Iris or ciliary body mass ${ }^{a}$ & Negative & Negative & Negative & Negative \\
\hline \multicolumn{5}{|l|}{ Imaging features } \\
\hline UBM & Indistinct & Indistinct & Indistinct & Indistinct \\
\hline \multicolumn{5}{|l|}{ AS-OCT } \\
\hline Visualization & Partial $^{\mathrm{b}}$ & Partial $^{\mathrm{b}}$ & Partial $^{\mathrm{b}}$ & Partial $^{\mathrm{b}}$ \\
\hline \multicolumn{5}{|l|}{ IVFA } \\
\hline Visualization & NA & NA & NA & Yes \\
\hline Vascular course & NA & NA & NA & PI-Pupil-PI \\
\hline Hemangioma type & NA & NA & NA & Simple \\
\hline \multicolumn{5}{|l|}{ OCTA } \\
\hline Visualization & Yes & Yes & Yes & Yes \\
\hline Vascular course & PI-Pupil-PI & PI-Pupil-PI & PI-Midzone-PI & PI-Pupil-PI \\
\hline Hemangioma type & Simple & Complex & Simple & Simple \\
\hline
\end{tabular}

jamaophthalmology.com
JAMA Ophthalmology
Abbreviations: AS-OCT, anterior segment optical coherence tomography; IVFA, intravenous fluorescein angiography; NA, not applicable/not done; OCTA, optical coherence tomography angiography; $\mathrm{PI}$, peripheral iris; UBM, ultrasound biomicroscopy.

a Imaged using UBM and AS-OCT.

b Lumen of racemose hemangioma was partially visualized. 
Figure 1. Iris Racemose Hemangioma Under Slitlamp Photographs (A, C, and E) and Corresponding Optical Coherence Tomography Angiography ( $B, D$, and F)

A Slitlamp photograph B Optical coherence tomography angiography
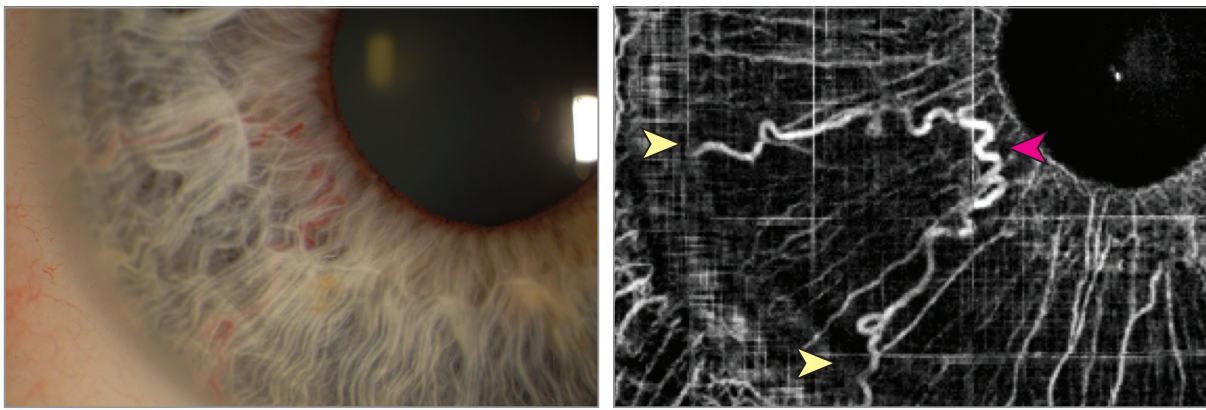

C Slitlamp photograph

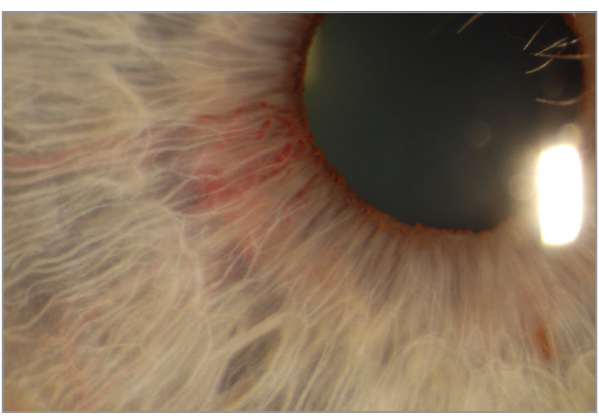

D Optical coherence tomography angiography

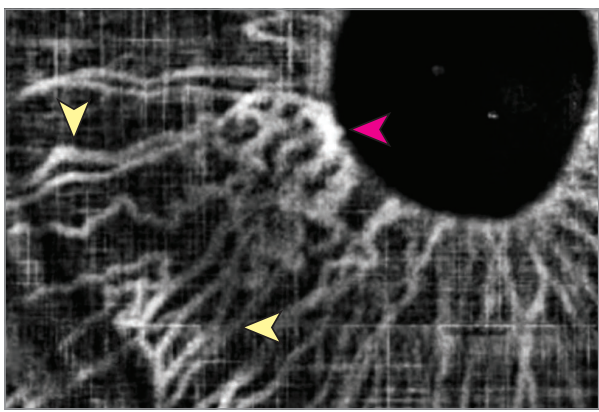

E Slitlamp photograph

F Optical coherence tomography angiography
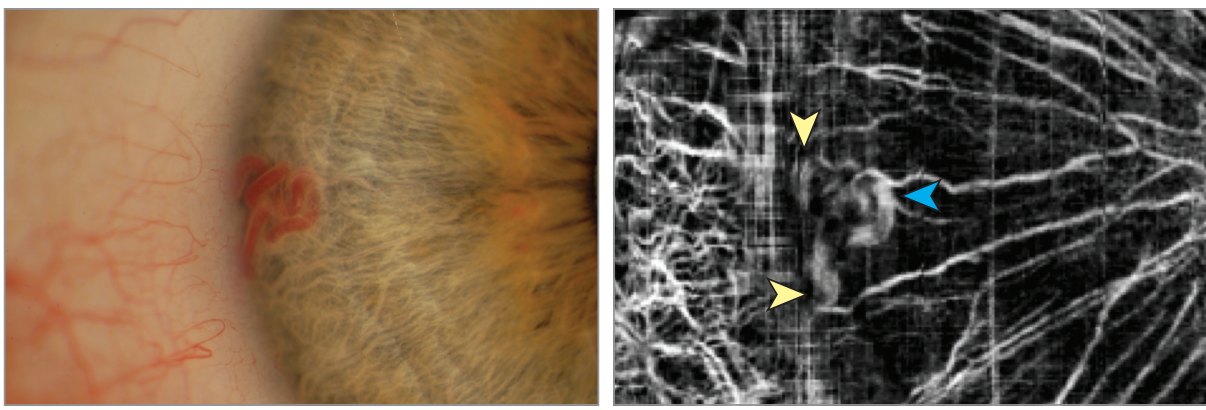

Optical coherence tomography angiography showed all iris racemose hemangiomas to be of uniform vascular caliber with a consistent looping course extending from the peripheral iris (yellow arrowheads) to the pupillary margin (red arrowhead) or midzonal iris (blue arrowhead). ize vascular details in the angle; hence, it was difficult to ascertain the exact origin of the hemangioma by OCTA. In the 1 case that received AS IVFA, the vascular course of the hemangioma was delineated on AS IVFA; however, details regarding relationship to normal iris microvasculature were not fully captured on AS IVFA (Figure 2B and C).

\section{Discussion}

Optical coherence tomography angiography can provide vascular imaging capability of iris and conjunctival tumors. ${ }^{6-8}$ Skalet et $\mathrm{al}^{6}$ showed increased intratumoral vascular density in iris melanomas compared with benign iris nevi using AS OCTA. Kang et $\mathrm{al}^{7}$ demonstrated iris microhemangiomatosis by OCTA, delineating pinpoint vascular lesions with flow arising from the posterior iris stroma, while Chien et $\mathrm{al}^{8}$ used OCTA to visualize abundant vasculature within conjunctival racemose hemangioma not seen on IVFA.

In this study, we found that commercially available OCTA showed remarkable clinical utility for imaging iris racemose hemangioma. Cases in this study had lightly pigmented irides. In the iris, detection of flow signal depends on iris color, and flow is best detected in lightly colored irides. ${ }^{6}$ Thus, our findings may not be replicable in darkly pigmented irides. Further, OCTA allowed detailed visualization of the hemangioma in relation to the normal iris vasculature not possible with slitlamp examination, particularly when they were deep within the iris stroma as demonstrated in patients 1,2 , and 4 . For patient 4 , OCTA provided visualization of the hemangioma superimposed on the normal radial iris vessels, while the normal radial iris vessels were not distinctly visualized on IVFA. This is likely owing to the long operating wavelength of OCTA, allowing better 
Figure 2. Iris Racemose Hemangioma Under Slitlamp Photograph, Early-Phase and Late-Phase Intravenous Fluorescein Angiography, and Optical Coherence Tomography Angiography
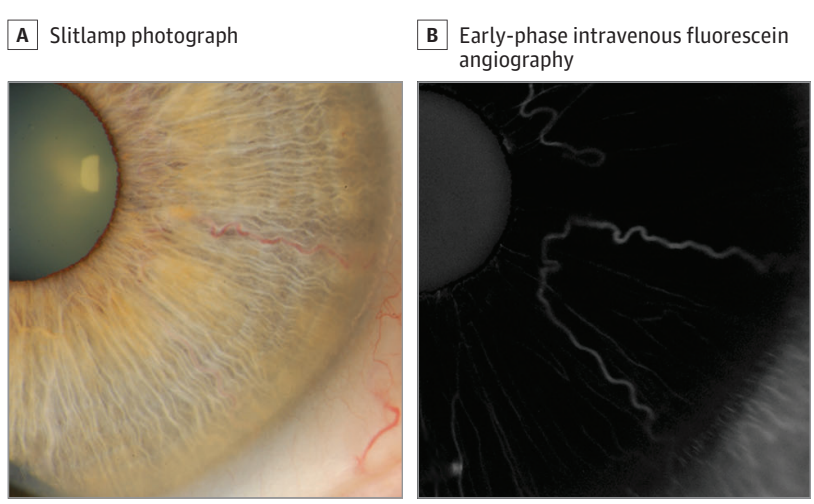

Optical coherence tomography angiography (D) showed dilated tortuous hyperreflective vascular lesion consistent with iris racemose hemangioma with looping course of vessel from the peripheral iris (yellow arrowhead) toward the pupillary margin (red arrowhead) and back to the peripheral iris (yellow

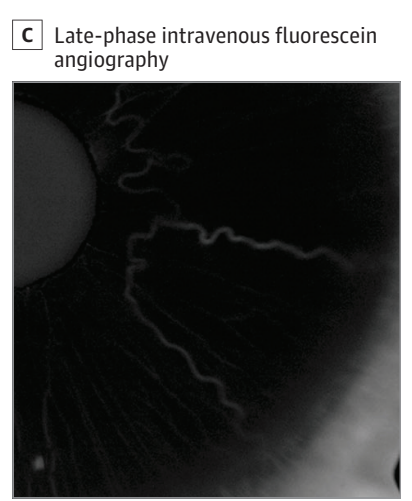

D Optical coherence tomography angiography

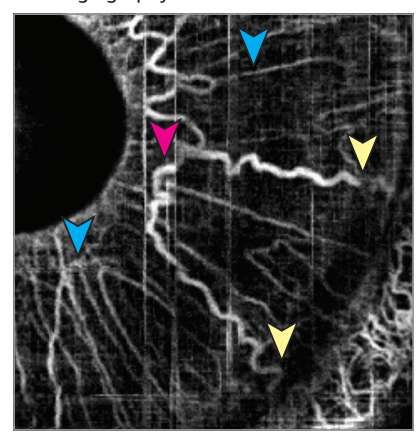

arrowhead). Small-caliber normal radial iris vessels (blue arrowhead) were more distinct on optical coherence tomography angiography (D) than early (B; 35 seconds) and late ( $C_{i}$ 2:05 minutes) phase intravenous fluorescein angiography. stromal tissue penetration, while IVFA exhibits diminished transmission of fluorescence through iris stroma.

Differentiation of iris racemose hemangioma from other vascular lesions is important. Iris racemose hemangioma is the most frequent iris vascular tumor and can be distinguished by its characteristic uniform vascular pattern, looping course, and location. ${ }^{2,10}$ In this study, OCTA showed racemose hemangioma emanates from the peripheral iris and courses to the pupillary margin or midzonal region, forming a tortuous loop before returning to the peripheral iris. In contrast, iris microhemangiomatosis is a minute nodular vascular sac at the pupillary margin, producing transient and intermittent hyphema, ${ }^{10-12}$ while iris neovascularization appears as fine, thread-like haphazard vascularization of the iris stroma emanating from the pupillary margin or the angle. ${ }^{10,13}$

\section{Limitations}

The characteristic vascular pattern of iris racemose hemangioma was identified on both OCTA and traditional IVFA. However, it is important to realize several limitations of each method and that each should not be used interchangeably. Intravenous fluorescein angiography is limited by light penetrance of deeper tissue and vascular leakage that can obscure microvascular details. Conversely, OCTA is limited by motion artifact and its inability to detect some flow patterns, especially vascular leakage. In cases where iris vascular lesions are clini- cally indistinguishable, features on IVFA could potentially allow differentiation because iris racemose hemangioma does not leak, while iris neovascularization typically exhibits slow leakage of dye into the aqueous. ${ }^{10,13}$ In terms of time efficiency, OCTA may be advantageous because it allows a quicker noninvasive evaluation of iris microvasculature. Further, only lightly pigmented irides were imaged using commercially available OCTA. Light penetrance using superluminescent diode typically used in spectral domain OCT may not be sufficient for darker irides. Also, all patients scanned in this series had excellent cooperation during image acquisition. Anterior segment OCTA imaging requires patient cooperation because eye tracking and motion correction used for posterior segment OCTA demonstrate less proficiency when applied to the AS.

\section{Conclusions}

In summary, we report OCTA features in 4 cases of iris racemose hemangioma. Optical coherence tomography angiography allowed visualization of the characteristic uniform vascular caliber and looping course of iris racemose hemangioma and provided information in relation to normal iris microvasculature. Future improvements in OCTA technology may allow improved details and extend limitations on iris color.

\section{ARTICLE INFORMATION}

Accepted for Publication: July 27, 2017.

Published Online: September 14, 2017. doi:10.1001/jamaophthalmol.2017.3390

Author Contributions: Dr Shields had full access to all the data in the study and takes responsibility for the integrity of the data and the accuracy of the data analysis.

Concept and design: Chien, Sioufi, Say, Shields.

Acquisition, analysis, or interpretation of data: All authors.

Drafting of the manuscript: Chien, Sioufi.
Critical revision of the manuscript for important intellectual content: All authors. Obtained funding: Shields.

Administrative, technical, or material support: Ferenczy, Say, Shields.

Supervision: Ferenczy, Say, Shields.

Conflict of Interest Disclosures: All authors have completed and submitted the ICMJE Form for Disclosure of Potential Conflicts of Interest and none were reported.
Funding/Support: Support provided by Eye Tumor Research Foundation, Philadelphia, Pennsylvania (Dr Shields).

Role of the Funder/Sponsor: The funder had no role in the design and conduct of the study, collection, management, analysis, and interpretation of the data, preparation, review, or approval of the manuscript, and decision to submit the manuscript for publication. 


\section{REFERENCES}

1. Shields CL, Kancherla S, Patel J, et al. Clinical survey of 3680 iris tumors based on patient age at presentation. Ophthalmology. 2012;119(2):407-414

2. Shields JA, Streicher TF, Spirkova JH, Stubna M, Shields CL. Arteriovenous malformation of the iris in 14 cases. Arch Ophthalmol. 2006;124(3):370-375.

3. Lee BJ, Jeng BH, Singh AD. OCT and ultrasound biomicroscopic findings in iris arteriovenous malformation. Ophthalmic Surg Lasers Imaging. 2008;39(5):426-428.

4. Ang M, Sim DA, Keane PA, et al. Optical coherence tomography angiography for anterior segment vasculature imaging. Ophthalmology. 2015;122(9):1740-1747.

5. Ang M, Cai Y, MacPhee B, et al. Optical coherence tomography angiography and indocyanine green angiography for corneal vascularisation. Br J Ophthalmol. 2016;100(11):15571563.

6. Skalet $A H, L i Y, L u C D$, et al. Optical coherence tomography angiography characteristics of iris melanocytic tumors. Ophthalmology. 2017;124(2): 197-204.

7. Kang AS, Welch JR, Sioufi K, et al. Optical coherence tomography angiography of iris microhemangiomatosis. American Journal of Ophthalmology Case Reports. 2017;6:24-26. doi:10 .1016/j.ajoc.2017.02.003

8. Chien JL, Sioufi K, Shields CL. Optical coherence tomography angiography of conjunctival racemose hemangioma. Ophthalmology. 2017;124(4):449.

9. Jia Y, Tan O, Tokayer J, et al. Split-spectrum amplitude-decorrelation angiography with optical coherence tomography. Opt Express. 2012;20(4): 4710-4725.
10. Shields JA, Bianciotto C, Kligman BE, Shields $\mathrm{CL}$. Vascular tumors of the iris in 45 patients: the 2009 Helen Keller Lecture. Arch Ophthalmol. 2010; 128(9):1107-1113.

11. Dahlmann AH, Benson MT. Spontaneous hyphema secondary to iris vascular tufts. Arch Ophthalmol. 2001;119(11):1728.

12. Goyal S, Foster PJ, Siriwardena D. Iris vascular tuft causing recurrent hyphema and raised IOP: a new indication for laser photocoagulation, angiographic follow-up, and review of laser outcomes. J Glaucoma. 2010;19(5):336-338.

13. Gartner S, Henkind P. Neovascularization of the iris (rubeosis iridis). Surv Ophthalmol. 1978;22(5): 291-312. 